\title{
Hydroxyapatite Thin Films Dedicated to Medical Applications
}

\author{
Predoi D1, Ghita RV1*, Iconaru SL ${ }^{1}$ and Beuran M$^{2}$ \\ ${ }^{1}$ National Institute of Materials Physics, Magurele, Romania \\ ${ }^{2}$ Carol Davila University of Medicine and Pharmacy, Romania
}

*Corresponding author: Ghita RV, National Institute of Materials Physics, Atomistilor 405 A, 077125, Magurele, Romania, Tel: 0723199 397; Email:

\author{
Mini Review
Volume 4 Issue 2
Received Date: April 24, 2019
Published Date: May 13, 2019
DoI: $10.23880 /$ nnoa-16000160
}

ghitar@infim.ro

\section{Abstract}

One of the major problems encountered in modern medicine is the finding of new materials that can be integrated in the human body without rejection by the host tissue. In this regard, all over the world the research was focused on the development of novel hybrid materials by combing polymer science and nanotechnology for different biomedical applications, such as drug delivery or biomimetic implants. For a successfully integration in human body these nanoengineered materials must have specific properties as good biocompatibility and biodegradability.

Keywords: Hydroxyapatite; Medicine; Materials; Nanotechnology; Thin Films

Abbreviations: HAP: Hydroxyapatite; PDMS: Polydimethylsiloxane; UV: ultraviolet; XPS: X-ray Photoelectron Spectroscopy; GDOES: Glow Discharge Optical Emission Spectroscopy; CLSM: Confocal Laser Scanning Microscopy; FTIR: Fourier transform infrared spectroscopy; S.aureus: Staphylococcus aureus; E.coli: Escherichia coli; CFU: Colony forming units.

\section{Mini Review}

Synthetic hydroxyapatite (HAp) with chemical formula $\mathrm{Ca}_{10}\left(\mathrm{PO}_{4}\right)_{6}(\mathrm{OH})_{2}$, belonging to the family of calcium phosphate ceramics is a good candidate for various biomedical applications. Due to its similarity to the mineral phase of human bone tissue and its outstanding properties, such as biocompatibility, bioactivity, osteoconductivity, porosity and long degradation time, it has been extensively used in orthopedic surgery as coating material for hip and knee prosthesis or for bone reconstruction, as well as in dentistry as filler in dental prosthesis [1]. In order to improve the biological properties of HAp, different polymers can be incorporated in its matrix together with different dopant elements such as Silver (Ag). It is well known that silver exhibits high antimicrobial activity against a large number of bacterial and fungal strains. In this regard, silver nanoparticles attach to the bacterial cellular membrane causing modification of its permeability and thus affecting the respiratory function. One of polymer studied in HAp matrix is polydimethylsiloxane (PDMS) -a member of organosilicon compounds exhibiting properties e.g. high thermal, ultraviolet (UV) and oxidative stability, low surface energy, hydrophobicity or physiological inertness, that recommend it to a good candidate to improve HAp mechanical properties. One of the technique that can be used in depositing Ag-NPs:HAp-PDMS/Si thin films is controlled thermal evaporation in medium vacuum (p $\sim \bullet \cdot 10^{-5}$ torr, deposition cycle: $120 \mathrm{~min}$, evaporation temperature $1100^{\circ} \mathrm{C}$ ). The X-ray Photoelectron Spectroscopy (XPS) analysis applied to Ag:HAp-PDMS/Si thin films generally reveals the formation of $\mathrm{SiO}_{4}{ }^{4-}$ ions incorporated in matrix by substitution of $\mathrm{PO}_{4}{ }^{3-}$ ions together with the structure of Ag:HAp. The Glow Discharge Optical Emission Spectroscopy (GDOES) analysis on thin films indicates that the composite layer is 


\section{Nanomedicine \& Nanotechnology Open Access}

homogeneous explained by the formation of Si-O-Ag and respectively Si-O-P bonds. For anti-biofilm activity the experiments were carried out on the obtained composite against Candida albicans- exposed because the fungal infections represent an emerging medical problem. In this regard, the design of novel biomaterials overcome a challenging problem that of the biofilm resistance of phenotype type in order to improve resistance to microbial colonization. The effect of C.albicans biofilm development on different substrates presents a significant decrease on Ag:HAp-PDMS composite layer as can be observed in Figure 1 [1].

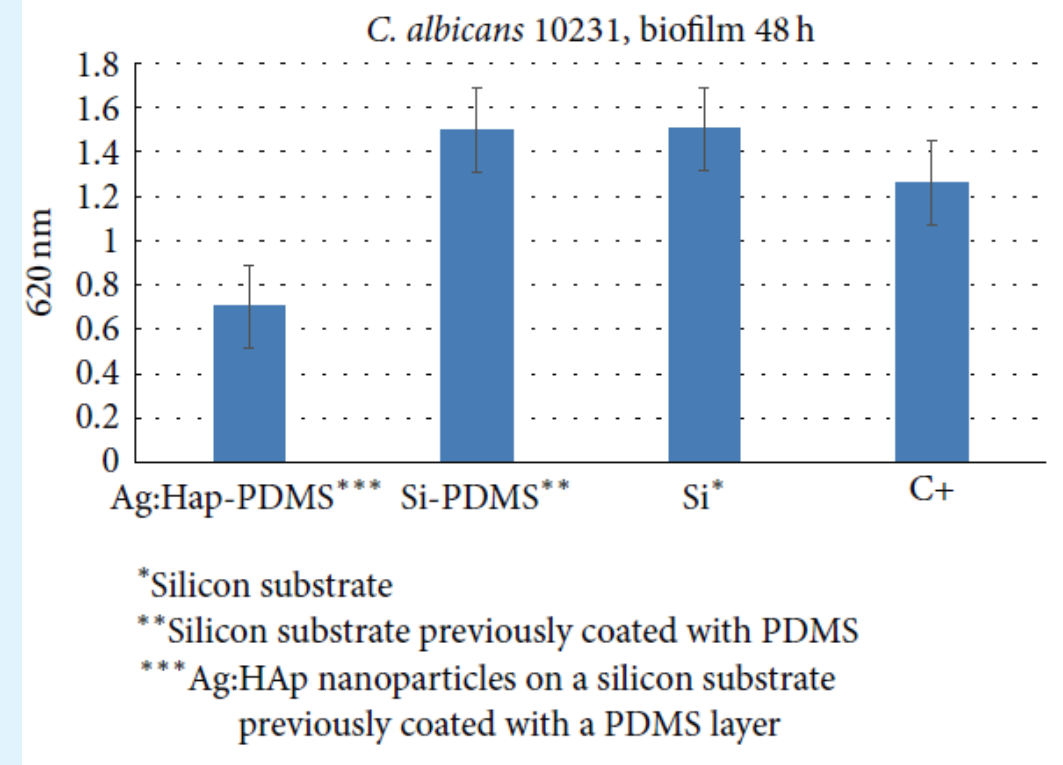

Figure 1: Representation of fungal biofilm development on different substrates.

It is important to remark that an important feature of hydroxyapatite structure is its permissiveness for different ionic substitutions, and in this regard there will be presented some data on Sm:HAp thin films [2]. Samarium is a rare-earth element with different nuclear applications, but from the biological point of view, the benefic role of $\mathrm{Sm}$ is related to the one of the tree radionuclides currently used for treating bone pain associated with different types of advanced cancer. The Sm:HAp thin films were obtained by controlled thermal evaporation in medium vacuum and from the morphological point of view the SEM and Confocal Laser Scanning Microscopy (CLSM) techniques reveal an uniform and homogeneous deposition in as presented experimentally conditions. Combination of Samarium and Hydroxyapatite could be used in medical applications and from this point of view there exist data related to antifungal activity that put into evidence that $\mathrm{Sm}: \mathrm{HAp} / \mathrm{Ti}$ had a fungicidal effect against C.albicans biofilm formation.

An important part for bioactivity of Ag:HAp is related to antibacterial activities of this material and various types of antibiotics such as tetracycline (T-HAp and T$\mathrm{Ag}: \mathrm{HAp}$ ) or ciprofloxacin (C-HAp and C-Ag:HAp) [3], where it is combined the biocompatible properties of HAp with the effects of well-known antibiotics as well as with the natural effect effects of silver ions. The SEM morphological studies on as prepared thin films indicates a granular shape influenced by the antibiotics and moreover, the elemental mapping showed a uniform distribution of elements on the entire film surface. In Fourier transform infrared spectroscopy (FTIR) analysis were identified the principal vibrational bands in the films associated with apatite structure with slightly adulterated by the presence of antibiotics. The GDOES studies indicated that in the inner structure of the film, the antibiotics were successfully incorporated. The bactericidal properties of HAp, T-HAp, Ag:HAp, T-Ag:HAp, C-HAp and C-Ag:HAp were evaluated using Staphylococcus aureus (S.aureus 0364) and Escherichia coli (E.coli ATCC 25922) a model for Gram-positive and Gram-negative strains. The newly created materials based on HAp and Ag:HAp and antibiotics provided a strong bactericidal effect against S.aureus 0364 and a reduction of CFU 


\section{Nanomedicine \& Nanotechnology Open Access}

(Colony forming units) number after $6 \mathrm{~h}$ of exposure to E.coli ATCC 25922 which was caused by the bacteriostatic effect, as can be observed in Figure 2 [3]. The results suggests that the effect of combining HAp and Ag:HAp with some of the most used antibiotics in a number of bacterial infections can produce a powerful composite capable of eradicating various bacterial strains.

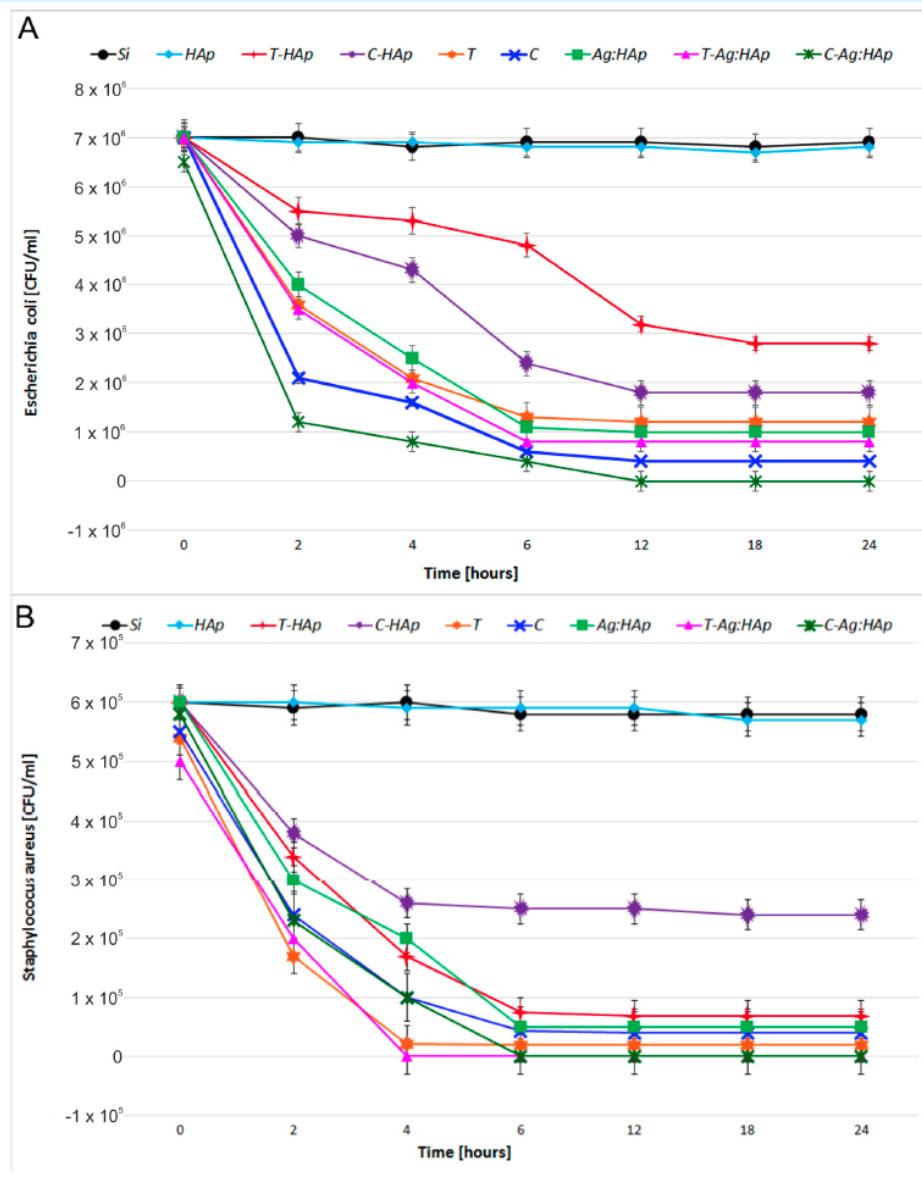

Figure 2: Bactericidal effects of time of contact with the surface of HAp, T-HAp, C-HAp, Ag:HAp, T-Ag:HAp, C-Ag:HAp, C and T thin films for E.coli ATCC 25922 (A) and S.aureus 0364 (B)

For current medical practice in different medical fields one problem encountered is the apparition of implantrelated infections and this problem conduced to solutions in developing new implants less susceptible to infections [4]. The most common microbial strains involved in implants related infections are staphylococci with nosocomial pathogen S.aureus and fungal pathogen C.albicans, both of them known for their ability to form biofilm, as an important condition to the development of implant related infections. In this condition our effort was focused on the development of biocompatible coatings with antifungal properties on titanium substrates. In this perspective, there was experienced spin-coating technique to prepare Ag:HAp composite layers from stable gels, where the characteristics e.g. stability of synthesized gels were evaluated by ultrasonic measurements. In ultrasonic method different characteristics as particle size and stability are correlated to ultrasound attenuation in gel suspension, e.g. in ultrasound dynamic regime. The experimental route for preparing Ag:HAp layers include sol-gel dip coating method deposition on pure Ti substrate using complex reagents like calcium nitrate $\mathrm{Ca}\left(\mathrm{NO}_{3}\right)_{2} \bullet 4 \mathrm{H}_{2} \mathrm{O}$, triethyl phosphite (TEP) $\mathrm{P}\left(\mathrm{C}_{2} \mathrm{H}_{5} \mathrm{O}\right)_{3}$ and silver nitrate $\mathrm{AgNO}_{3}$, together with a thermal treatment for drying and an annealing for $12 \mathrm{~h}$ at $300^{\circ} \mathrm{C}$ [4]. The gels characteristics 


\section{Nanomedicine \& Nanotechnology Open Access}

were investigated by ultrasound measurements due to its major advantage of wave propagation through concentrated opaque suspensions and to the possibility to extract information regarding the stability of resulted gel. In ultrasound method there were extracted information regarding suspension concentration from data related to stability parameter and time parameter of first period, as a stability indicator. The as obtained bioceramic layers of $\mathrm{Ag}: \mathrm{HAp} / \mathrm{Ti}$ (Figure 3)[4] can induce the proliferation of osteoblasts and the CLMS analysis put into evidence a strong antifungal activity against C.albicans ATC 10231 and completely inhibited the biofilm formation.



Figure 3: SEM image of Ag:HAp composite layer

The present study put into evidence the effort of complex biomaterial preparation as synthetic HAp thin films obtained by different deposition methods on various substrates, doped with different elements in order to enhance biological activity. Hydroxyapatite is viewed as a biomaterial where the biocompatible characteristics can be combined with effects of known antibiotics to produce a composite capable to eradicated various bacterial strain or in doped Ag:HAp composite layers there exist an intense manifestation of antifungal activity. The research effort is focused on the development of bioengineering in order to obtain new and improved biomaterials to be used in different biomedical applications.

\section{References}

1. Ciobanu CS, Groza A, Iconaru SL, Popa CL, Chapon P, et al. (2015) Antimicrobial Activity Evaluation on
Silver Doped Hydroxyapatite/Polydimethylsiloxane Composite Layer. Bio Med Research International.

2. Iconaru SL, Stanciu GA, Hristu R, Ghita RV (2017) Properties of Samarium Doped Hydroxyapatite Thin Films Deposited by Evaporation Romanian Reports in Physics 69.

3. Predoi D, Popa CL, Chapon P, Groza A, Iconaru SL (2016) Evaluation of the Antimicrobial Activity of Different Antibiotics Enhanced with Silver-Doped Hydroxyapatite Thin Films. Materials 9(9): 778.

4. Predoi D, Iconaru SL, Predoi MV (2018) Bioceramic Layers with Antifungal Properties. Coatings 8(8): 276. 